\title{
LETTER
}

\section{Frailty index predicts poor outcome in COVID-19 patients}

\author{
Giuseppe Bellelli ${ }^{1,2^{*}}$, Paola Rebora ${ }^{3}$, Maria Grazia Valsecchi ${ }^{3}$, Paolo Bonfanti ${ }^{1,4}$ and Giuseppe Citerio ${ }^{1}$ on behalf \\ of COVID-19 Monza Team members
}

๑ 2020 Springer-Verlag GmbH Germany, part of Springer Nature

Frailty is a condition of increased vulnerability to endogenous and exogenous stressors, resulting from the interaction of progressive age-related decline in physiologic systems with chronic diseases, leading to decreased functional reserve capacities [1]. The effect of frailty on patient's clinical outcomes has been examined in several settings of care, including intensive care units (ICU) and acute hospital wards $[1,2]$, showing to be a reliable predictor of clinical and health care related outcomes. Based on these and other evidences, some scientific societies (https://www.nice.org.uk/guidance/ng159/resources/criti cal-care-admission-algorithm-pdf-8708948893; https:// www.brc-rea.be/wp-content/uploads/2020/03/Ethicaldecision-making-in-emergencies_COVID19_22032020_ final.pdf) are recommending to assess frailty in patients with coronavirus disease 19 (COVID-19) infection to guide their triage. However, not all international scientific associations have similar positions (https://smw.ch/ article/doi/smw.2020.20229; http://www.siaarti.it/SiteA ssets/News/COVID19\%20-\%20documenti\%20SIAARTI/ SIAARTI\%20-\%20Covid 19\%20-\%20Rac comandazio ni\%20di\%20etica\%20clinica.pdf).

Therefore, we decided to evaluate the role of frailty assessment in patients with COVID-19. Here, we analyzed the data of a cohort of consecutive COVID-19 patients admitted to 8th floor of San Gerardo hospital between February 27th and April 7th, 2020. Inclusion criteria were age $>18$ years, informed consent and hospitalization due to COVID-19 infection. There were no exclusion criteria. Frailty was assessed with the Frailty

*Correspondence: giuseppe.bellelli@unimib.it

1 School of Medicine and Surgery, University of Milano - Bicocca, Milan, Italy

Full author information is available at the end of the article
Index (FI), a commonly used tool which is based on the concept that frailty is the result of an accumulation of deficits during lifetime [3]. The FI evaluated coexisting diseases, cognitive and physical impairments and laboratory abnormalities. For each variable, we assigned a score 0 in the absence and 1 in the presence of a deficit. The score was calculated for each participant by dividing the sum of the deficits by the total number of variables measured. Overall, we assessed 43 variables, which provided our index with a sufficient amount of robustness [3]. Importantly, one study has shown that a FI constructed with a similar number of variables was superior to other frailty tools in predicting mortality [4]. The variables included in the 43-item FI are listed in the Electronic Supplementary Material along with the graphical distribution of the scores.

Among 105 patients included in our study, 40 had a "do not intubate" indication, 58 had an "intubate if needed" indication and 7 had neither. The FI median score was 0.17 [interquartile ranges, IQR 0.12, 0.26] among the 42 patients died or transferred to ICU and 0.07 [IQR 0.05, 0.14] among the 63 patients who recovered $(p<0.001)$ (Table 1). According to a previous study, participants with a FI score $\geq 0.25$ were considered frails [5]. In a multivariable logistic model (see Electronic Supplementary Material), including age, sex and FI, age and the dichotomized FI were independent predictors of inhospital mortality or ICU admission (odds ratio in patients with $\mathrm{FI} \geq 0.25$ vs $<0.251 .32$, 95\% confidence intervals: $1.03 ; 1.70$ ).

We suggest integrating the frailty assessment in all the COVID-19 patients at hospital admission. With electronic medical records progressively more available in the hospitals, the assessment of frailty with an electronic FI can help clinicians in their decision-making

\section{Springer}


Table 1 Baseline characteristics of patients by outcome (recovered or death/transferred to ICU)

\begin{tabular}{|c|c|c|c|}
\hline & Patients who recovered $(n=63)$ & $\begin{array}{l}\text { Patients who died or were transferred } \\
\text { to ICU }(n=42)\end{array}$ & $p$ value* \\
\hline Males, $n$ (\%) & $41(65)$ & $31(74)$ & 0.466 \\
\hline Age, years & $58.74[51.78,68.54]$ & $77.25[68.42,83.59]$ & $<0.001$ \\
\hline \multicolumn{4}{|l|}{ Diseases } \\
\hline Hypertension, $n(\%)$ & $33(52)$ & $31(74)$ & 0.045 \\
\hline Coronary heart disease, $n(\%)$ & $5(8)$ & $20(48)$ & $<0.001$ \\
\hline Atrial fibrillation, $n(\%)$ & $2(3)$ & $9(21)$ & 0.008 \\
\hline Peripheral vascular, n (\%) & $2(3)$ & $14(33)$ & $<0.001$ \\
\hline Congestive heart failure, $n(\%)$ & $1(2)$ & $3(7)$ & 0.349 \\
\hline Previous stroke, $n(\%)$ & $1(2)$ & $3(7)$ & 0.349 \\
\hline Diabetes, $n(\%)$ & $7(11)$ & $14(33)$ & 0.011 \\
\hline Chronic respiratory, $n$ (\%) & $2(3)$ & $4(10)$ & 0.345 \\
\hline Chronic renal failure, $n(\%)$ & $5(8)$ & $2(5)$ & 0.811 \\
\hline Liver, $n(\%)$ & $4(6)$ & $1(2)$ & 0.64 \\
\hline Altered thyroid function, $n(\%)$ & $6(9.5)$ & $2(4.8)$ & 0.599 \\
\hline Osteoarthritis, $n(\%)$ & $1(2)$ & $1(2)$ & 1 \\
\hline Osteoporosis, $n(\%)$ & $0(0)$ & $3(7)$ & 0.12 \\
\hline Solid neoplasm, $n$ (\%) & $7(11)$ & $4(10)$ & 1 \\
\hline Lymphoma/leukemia, n (\%) & $0(0)$ & $2(5)$ & 0.308 \\
\hline Peptic ulcer, $n(\%)$ & $1(2)$ & $1(2)$ & 1 \\
\hline Rheumatic, $n(\%)$ & $4(6)$ & $4(10)$ & 0.822 \\
\hline Anemia, $n(\%)$ & $2(3)$ & $1(2)$ & 1 \\
\hline Hearing impairment, $n$ (\%) & $1(2)$ & $2(5)$ & 0.72 \\
\hline Visual impairment, $n(\%)$ & $4(6)$ & $3(7)$ & 1 \\
\hline Depressed mood, $n(\%)$ & $3(5)$ & $1(2)$ & 0.917 \\
\hline Dementia, $n(\%)$ & $3(5)$ & $2(5)$ & 1 \\
\hline Parkinson/parkinsonism, $n$ (\%) & $0(0)$ & $2(5)$ & 0.308 \\
\hline Nutritional status & & & 0.018 \\
\hline Undernourished, $n(\%)$ & $0(0)$ & $2(7)$ & \\
\hline Normal, $n(\%)$ & $48(89)$ & $19(66)$ & \\
\hline Obese, $n(\%)$ & $6(11)$ & $8(28)$ & \\
\hline Number of drugs & $2[1,3.75]$ & $8[2,10]$ & $<0.001$ \\
\hline \multicolumn{4}{|l|}{ Disability } \\
\hline Self-doing in bathing, $n$ (\%) & $4(7)$ & $9(27)$ & 0.014 \\
\hline Self-dressing, $n(\%)$ & $3(5)$ & $6(20)$ & 0.062 \\
\hline Walking at home, $n(\%)$ & $3(5)$ & $5(17)$ & 0.135 \\
\hline Walking out of home, $n(\%)$ & $4(6)$ & $7(23)$ & 0.046 \\
\hline Shopping, $n(\%)$ & $5(8)$ & $10(32)$ & 0.009 \\
\hline Unable to drive a car, $n$ (\%) & $8(13)$ & $13(43)$ & 0.004 \\
\hline Unable to handle money, $n(\%)$ & $6(10)$ & $7(23)$ & 0.168 \\
\hline Unable to handle drugs, $n(\%)$ & $6(10)$ & $9(30)$ & 0.036 \\
\hline Nursing home resident/caregiver, $n(\%)$ & $2(3)$ & $4(12)$ & 0.234 \\
\hline \multicolumn{4}{|l|}{ Laboratory findings (serum levels) } \\
\hline Hemoglobin g/dl & $13.50[12.1,15]$ & $13.65[11.62,15.05]$ & 0.554 \\
\hline White blood cell count, $\times 10^{9}$ & $5.78[4.37,7.32]$ & $6.74[5.1,9.45]$ & 0.065 \\
\hline Lymphocites count, $\times 10^{9}$ & $1.13[0.89,1.47]$ & $0.88[0.6,1.16]$ & 0.024 \\
\hline Platelet count, $\times 10^{9}$ & $182[160,219]$ & $176.5[146.25,252.75]$ & 0.702 \\
\hline Lactate dehydrogenase, $\mathrm{U} / \mathrm{L}$ & $292[247,365]$ & $367[290.25,468.25]$ & 0.008 \\
\hline C-reactive protein, $\mathrm{mg} / \mathrm{dL}$ & $5.64[3.07,10.9]$ & $9.66[5.46,17.72]$ & 0.003 \\
\hline International normalized ratio & $1.09[1.03,1.16]$ & $1.15[1.06,1.27]$ & 0.071 \\
\hline
\end{tabular}


Table 1 (continued)

\begin{tabular}{|c|c|c|c|}
\hline & Patients who recovered $(n=63)$ & $\begin{array}{l}\text { Patients who died or were transferred } \\
\text { to ICU }(n=42)\end{array}$ & $p$ value* \\
\hline Creatine kinase, $U / L$ & $98[68.75,209]$ & $124.5[66,241.25]$ & 0.447 \\
\hline Albumin, g/dL & $3.11[2.96,3.4]$ & $3.2[3,3.54]$ & 0.685 \\
\hline Total bilirubin, $\mathrm{mmol} / \mathrm{L}$ & $0.6[0.3,0.7]$ & $0.5[0.4,0.8]$ & 0.212 \\
\hline Creatinine, mmol/L & $1[0.8,1.1]$ & $1.1[1,1.4]$ & 0.001 \\
\hline Frailty Index & $0.07[0.05,0.14]$ & $0.19[0.14,0.26]$ & $<0.001$ \\
\hline
\end{tabular}

Data are presented as median [Interquantiles range] unless otherwise specified

The score of the Frailty Index was based on the assessment of 43 health deficits. For each variable, we assigned a score 0 in the absence and 1 in the presence of a deficit. The score was calculated for each participant by dividing the sum of the deficits by the total number of variables measured

*Significance on Fisher exact test or Mann-Whitney test as appropriate

processes, identifying patients most likely to require ICU admission and those with poor outcomes. Future studies are needed to determine if FI is superior to other tools in predicting the outcomes of COVID-19 patients.

\section{Electronic supplementary material}

The online version of this article (https://doi.org/10.1007/s00134-020-06087-2) contains supplementary material, which is available to authorized users.

\section{Author details}

${ }^{1}$ School of Medicine and Surgery, University of Milano - Bicocca, Milan, Italy. ${ }^{2}$ Acute Geriatric Unit, San Gerardo Hospital, Monza, Italy. ${ }^{3}$ Bicocca Center of Bioinformatics, Biostatistics and Bioimaging, School of Medicine and Surgery, University of Milano - Bicocca, Monza, Italy. ${ }^{4}$ Infectious Diseases Unit, San Gerardo Hospital, Monza, Italy.

\section{Authors contribution}

All authors designed the study. GB and PM collected the clinical data. MGV and PR performed the statistical analyses. All authors reviewed and approved the manuscript.

\section{COVID-19 Monza Team members}

Stefania Galimberti, Bicocca Center of Bioinformatics, Biostatistics and Bioimaging, School of Medicine and Surgery, University of Milano - Bicocca, Monza, Italy; Paolo Blangiardo, MD, School of Medicine and Surgery, University of Milano - Bicocca, Monza, Italy; Gabriella Maria Celora, MD, School of Medicine and Surgery, University of Milano - Bicocca, Monza, Italy; Valentina Deiana, MD, School of Medicine and Surgery, University of Milano - Bicocca, Monza, Italy; Nives Ghezzi, MD, School of Medicine and Surgery, University of Milano - Bicocca, Monza, Italy; Alice Marchegiani, MD, School of Medicine and Surgery, University of Milano - Bicocca, Monza, Italy; Julia Miksza, MD, School of Medicine and Surgery, University of Milano - Bicocca, Monza, Italy; Andrea Piazzoli, MD, School of Medicine and Surgery, University of Milano - Bicocca, Monza, Italy.

\section{Funding}

None.

\section{Compliance with ethical standards}

\section{Conflicts of interest}

None.

\section{Ethical approval}

The study was approved by the institutional Ethical committee Brianza and was performed in adherence to the tenets of the Declaration of Helsinki.

\section{Consent to participate}

Informed consent was verbally obtained from all individuals participating in the study. Written consent was waived by Ethical Committee due to the context of emergency, which made hard circulating papers in the recruiting wards.

\section{Publisher's Note}

Springer Nature remains neutral with regard to jurisdictional claims in published maps and institutional affiliations.

Accepted: 4 May 2020

Published online: 25 May 2020

\section{References}

1. Clegg A, Young J, lliffe S, Rikkert MO, Rockwood K (2013) Frailty in elderly people. Lancet 381(9868):752-762

2. Muscedere J, Waters B, Varambally A et al (2017) The impact of frailty on intensive care unit outcomes: a systematic review and meta-analysis. Intensive Care Med 43:1105-1122

3. Searle SD, Mitnitski A, Gahbauer EA, Gill TM, Rockwood K (2008) A standard procedure for creating a frailty index. BMC Geriatr 8:24

4. Zucchelli A, Vetrano DL, Grande G et al (2019) Comparing the prognostic value of geriatric health indicators: a population-based study. BMC Med 17(1):185

5. Song X, Mitnitski A, Rockwood K (2010) Prevalence and 10-year outcomes of frailty in older adults in relation to deficit accumulation. J Am Geriatr Soc 58(4):681-687 